\title{
Responses of Spectrally Selective Cells in Macaque Area V2 to Wavelengths and Colors
}

\author{
K. MOUTOUSSIS AND S. ZEKI \\ Wellcome Department of Cognitive Neurology, University College London, London WC1E 6BT, United Kingdom
}

Received 23 March 2001; accepted in final form 28 November 2001

\begin{abstract}
Moutoussis, K. and S. Zeki. Responses of spectrally selective cells in macaque area V2 to wavelengths and colors. J Neurophysiol 87: $2104-2112$, 2002; 10.1152/jn.00248.2001. We have recorded from wavelength-selective cells in macaque monkey visual area V2, interposed between areas V1 and V4 of the color-specialized pathway, to learn whether their responses correlate with perceived colors or are determined by the wavelength composition of light reflected from their receptive fields. All the cells we recorded from were unselective for the orientation and direction of motion of the stimulus, and all were histologically identified to be in the thin cytochrome oxidase stripes. Using multi-colored "Mondrian" scenes of the appropriate spatial configuration, areas of different color were placed in the receptive field of each cell and the entire scene illuminated by three projectors, passing long-, middle-, and short-wave light, respectively, in various combinations. Our results show that wavelength-selective cells in V2 respond to an area of any color depending on whether or not it reflects a sufficient amount of light of their preferred wavelength. In addition, the responses of a third of the cells tested were also influenced by the wavelength composition of their immediate surrounds, thus signaling the result of a local spatial comparison with respect to the amount of their preferred wavelength present. The responses of all also depended on the sequence with which their receptive fields were illuminated with light of the three different wavebands: cells were activated when there was an increase (and inhibited when there was a decrease) in the amount of their preferred wavelength with respect to the other two; the temporal route taken was therefore a determining factor, and, depending on it, cells would either respond or not to a particular combination of wavelengths. We conclude that although spatiotemporal wavelength comparisons are taking place in the color-specialized subdivisions of area V2, the determination of complete color-constant behavior at the neuronal level requires further processing, in other areas.
\end{abstract}

\section{N T R O D U C T I O N}

Area V2 of the macaque brain is interposed between the primary visual cortex, area V1, and the other visual areas of the occipital lobe. The nature of visual field representation in it, as well as its extent and borders with V1 and V3, were first revealed by anatomical studies in the macaque monkey (Cragg 1969; Zeki 1969) and subsequently found to be true of all primates, including man (Allman and Kaas 1971; Sereno et al. 1995; Shipp et al. 1995). V2 contains cells with many different functional properties, including ones that are selective for color, orientation or direction of motion (see following text). Cytochrome oxidase staining shows that V2 consists of three

Address for reprint requests: S. Zeki, Wellcome Dept. of Cognitive Neurology, University College London, Gower St., London WC1E 6BT, UK (E-mail: zeki.pa@ucl.ac.uk). different types of alternating stripes, thick $(\mathrm{K})$, thin $(\mathrm{N})$, and inter (I) (Livingstone and Hubel 1982; Tootell et al. 1983). Most physiological studies agree that this characteristic architecture is the anatomical reflection of a functional segregation within V2 (see Fig. 1), with spectrally selective ("color") cells being concentrated in the thin stripes, directionally selective cells in the thick stripes, and orientation selective cells in both thick stripes and inter-stripes (Baizer et al. 1977; DeYoe and Van Essen 1985; Hubel and Livingstone 1985, 1987; Malach et al. 1994; Roe and Ts'o 1995; Shipp and Zeki 1985; Tootell and Hamilton 1989; Ts'o et al. 1990). Even studies that do not find a tight correlation between functional properties of cells and their anatomical groupings (DeYoe and Van Essen 1985; Gegenfurtner et al. 1996; Levitt et al. 1994) nevertheless show color cells to be more frequently found in the thin stripes, direction-selective cells in the thick stripes, and orientationselective cells in the thick stripes and interstripes and exclusively so if they occur in clusters. This functional segregation of color in V2 is also anatomically evident in this area's afferent and efferent connections: thin stripes receive their input from V1 cytochrome oxidase blobs, which are mainly driven by the parvocellular layers of the LGN and contain cells selective for the color of the stimulus (Born and Tootell 1991; Livingstone and Hubel 1983, 1984; Tootell et al. 1988; Ts'o and Gilbert 1988) and project mainly to V4 (DeYoe and Van Essen 1985; DeYoe et al. 1994; Felleman et al. 1997; Malach et al. 1994; Nakamura et al. 1993; Shipp and Zeki 1985; Xiao et al. 1999; Zeki and Shipp 1989), an area specialized in color processing (Zeki 1973, 1977, 1978a,b). The picture of functional specialization that emerges from past studies of V2 reflects, we believe, the more global functional specialization that characterizes the primate visual brain (for a review, see Zeki 2001). This is even reflected in the temporal dimension because different attributes of the visual scene are perceived asynchronously, color being perceived before motion and form (Moutoussis and Zeki 1997a,b). There is, however, an alternative view of the organization of the visual brain, with which we disagree, namely that all visual areas, far from being specialized, are in fact multi-purpose (for a review, see Schiller 1996, 1997).

Because V2 is an area that is anatomically and functionally interposed in the color pathway between the wavelength-selective cells in V1 and the color-selective cells in V4, we thought it interesting to learn whether its cells display the property of

\footnotetext{
The costs of publication of this article were defrayed in part by the payment of page charges. The article must therefore be hereby marked "advertisement" in accordance with 18 U.S.C. Section 1734 solely to indicate this fact.
} 
A

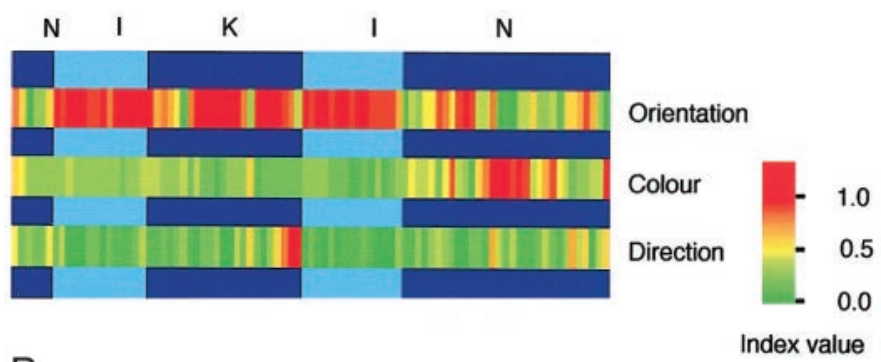

B

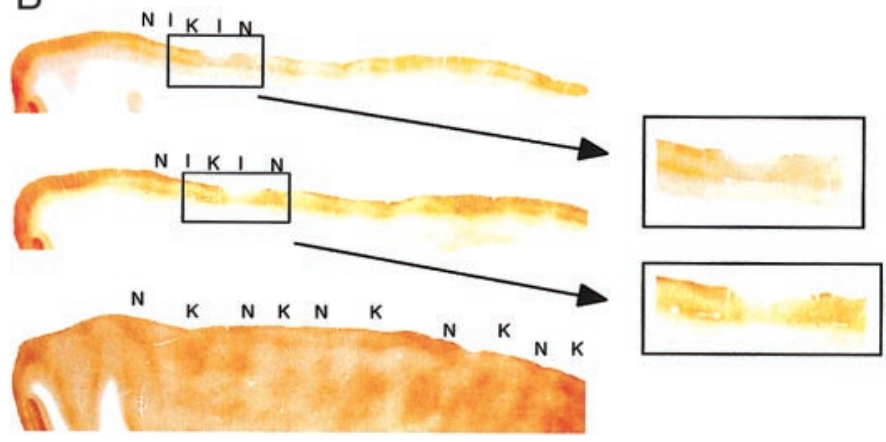

FIG. 1. A: schematic representation of orientation, color, and directional selectivities of cells along a single long penetration, perpendicular to the cytochrome oxidase (CO) stripes of V2. Recordings were made from 2 thin (N), 2 inter (I), and 1 thick (K) stripes. Every cell was given a selectivity index (DeYoe and Van Esssen 1985) for each 1 of the 3 visual attributes tested. The selectivity index varies from 0 to 1 (or higher if there is inhibition by 1 of the stimuli) and is color coded with red representing high selectivity and green no selectivity at all. The width of each stripe is representative of the number of cells recorded from it and does not reflect the actual size of the stripe in reality. $B$ : cytochrome-oxidase-stained sections of the flat-mounted operculum reconstructed in $A$. The entire stripe pattern is more clearly seen in the lower section; the stripes recorded from are shown in the 2 top sections, where the electrode track is present. A magnification of part of the recording sites is shown to the right, revealing parts of the electrode track and the lesions used as landmarks (see METHODS).

color constancy. Color constancy-the fact that the color of surfaces remains largely unaltered in spite of wide fluctuations in the wavelength/energy composition of the light reflected from them-is probably the most prominent characteristic of the color system. A possible strategy by which the brain achieves this is to compare the different amounts of long-, middle-, and short-wave light across space (Land 1974; Land and McCann 1971). Recent psychophysical and imaging evidence suggests that such a comparison is shared between early and late stages of the visual pathway (Bartels and Zeki 2000; Moutoussis and Zeki 2000). On the other hand, physiological evidence shows that cells whose responses correlate with color as perceived by humans, rather than the wavelength composition of the stimulus, occur in area V4 but not V1 (Zeki 1983a): striate cortex (and some V4) cells were found to respond to an area of any color in the scene, as long as it is made to reflect a sufficient minimum amount of light of their preferred wavelength and lesser amounts of the other two wavelengths. Some V4 cells, on the other hand, respond to an area of their preferred color irrespective of the wavelength composition of the light reflected from it and do not respond to areas of other colors even if they are reflecting light of the same wavelength composition as the area of their preferred color. We tried therefore to learn something about the role of cells in area V2 thin stripes in color processing and perception by testing whether they are genuine color cells, in the sense of responding to a patch of a given color irrespective of the wavelength composition of the light reflected from it, or whether they are only wavelength selective. If the latter, they should respond to a patch of any color if it reflects sufficient amounts of light of their preferred wavelength. In summary, we were interested to see whether V2 cells with a strong wavelength preference across the spectrum are able to discriminate between wavelength composition and real color, and able to compare between the various wavelength compositions across space and time.

\section{METHODS}

\section{Animal preparation}

Four male juvenile macaques between 12 and 20 mo of age were used. Each was first sedated with $20 \mathrm{mg} / \mathrm{kg}$ ketamine (Vetalar, ParkerDavis), cannulated via the saphenous vein, and intubated through the mouth. Propofol (Diprivan, Zeneca) was continuously perfused intravenously at a rate of $4-12 \mathrm{mg} \cdot \mathrm{kg}^{-1} \cdot \mathrm{h}^{-1}$ to maintain anesthesia. Sufentanil citrate (Janssen) and pancuronium bromide (Pavulon, Organon Technika) were also infused alongside propofol at the rates of 3-8 and $60 \mu \mathrm{g} \cdot \mathrm{kg}^{-1} \cdot \mathrm{h}^{-1}$, respectively. The monkey was respired using a Harvard respirator (tidal volume: $11.6 \mathrm{~cm}^{3} / \mathrm{kg}$ ), and the respiration rate was adjusted to maintain the end tidal $\mathrm{CO}_{2}$ between 3 and $4 \%$ as measured by a "Cardiocap" electroencephalogram (ECG)/ gas analyzer. The depth of anesthesia was continuously monitored by examination of the ECG, rectal temperature (maintained at $38^{\circ} \mathrm{C}$ with the aid of an electric blanket), and exhaled $\mathrm{CO}_{2}$. The animal's head was rigidly placed in a head holder, using ear bars and a mouthpiece, that was designed to offer no obstruction to the field of view. A cut was made through the skin, and, after retracting the skin and muscle layers, a hole was made in the skull using a dental drill to expose the dura at around $1.5 \mathrm{~mm}$ forward from the occipital crest to reveal the lunate sulcus. The dura mater was then cut, and an area of cortex roughly $1 \mathrm{~cm}$ in diameter was exposed. This exposure was covered in cooled $2 \%$ agar-in-saline solution. The eyelids were retracted with $10 \%$ phenylephrine drops (Richard Daniel and Son), the pupils were dilated using $1 \%$ atropine eye drops (Schering-Plough), and neutral contact lenses were placed over the eyes. Additional auxiliary lenses, the strength of which was determined by streak retinoscopy, were used to focus the eyes on a target screen $114 \mathrm{~cm}$ in front of the animal. Using a reversible ophthalmoscope, the foveas were identified and, by rotating the instrument through $180^{\circ}$, the center of gaze was marked on the tangent screen.

\section{Recording}

Extracellular recording of neuronal activity was done with low impedance ( $1 \mathrm{M} \Omega$ at $1 \mathrm{kHz}$ ) and 10 - to $12-\mu \mathrm{m}$ exposed-tip-length gold-platinum-plated tungsten-in-glass microelectrodes (Merrill and Ainsworth 1972) mounted in an impedance matching headstage on a microdrive system. The electrode was inserted in the part of V2 near the edge of the lunate sulcus, at an angle parallel to the V1/V2 border and the sulcus itself, and advanced in a medial to lateral direction, i.e., perpendicular to the cytochrome oxidase stripes, so as to record from many different stripes in a single penetration (see Fig. 1). The electrode was advanced by a Burleigh "Inchworm" microdrive attached to the micromanipulator. The signal was fed into a "Neurolog" NL 100 headstage preamplifier and then filtered using a notch filter with a high-pass section between 300 and $800 \mathrm{~Hz}$ and a low-pass section between 5 and $10 \mathrm{kHz}$ (NL 104 AC preamplifier, NL 125 filters, NL 200 spike trigger, NL 606 Latch counter). In this way, we were normally able to record from single cells, although in a few cases, we might have recorded from more than one neuron (see DISCUSSION). 
Spikes were displayed on a Tektronix RM 565 oscilloscope fitted with a type 3A9 amplifier and relayed over a loudspeaker. The signal was also used to drive a spike trigger, and the transistor transistor logic (TTL) signals produced were recorded by the stimulation computer. Using a spike discriminator after the amplification stage, recordings were made from individual spikes that were thresholded, converted to TTL pulses and then fed to the computer, where they were collected in 20-ms bins and saved. The spike record was displayed during the stimulation on another monitor, driven by a secondary processor card fitted inside the computer. The average spike rate for each condition was displayed graphically, and the period during which the stimulus was inside the cell's receptive field was highlighted. To identify individual tracks and locations within the tracks, electrolytic lesions were made by passing current, 5-10 $\mu \mathrm{A}$ tip positive for 5-10 s.

\section{Stimulation and data analysis}

Stimuli were generated on a microcomputer system (Amiga 2000 microcomputer, CBM) and presented on a 19-in Grundig BGC155 color monitor. The stimuli consisted of bars of variable length, width, color, and orientation. The background color could also be varied. The stimuli were controlled manually by use of a trackball, or automatically by the computer, in a sequence designed to test one or another attribute of the cell. The receptive field of the cell could be located using the manual control system, and the positions of the edges of the receptive field (excitatory region) were entered into the computer. This information was used in the automatic tests to center the stimulus on the receptive field and to determine the exact epoch of stimulation. As well as the stimulus/background characteristics, speed of motion (which could also be equal to 0 to test directionally selective cells for any orientation preference), stimulus duration, intertrial period (during which the screen was blank, i.e., uniform gray, black, or any other color used as the background color during stimulation), and number of repetitions could also be entered for the automatic computer controlled testing of a cell. This enabled a wide range of quantitative tests to be devised that, together with the operator's experience and qualitative testing, were used to fully characterize each cell. To give a value to a cell's selectivity for a particular attribute, we used a selectivity index $I=[1-$ (worst-base)/(best-base) $]$ that compares the best and worst responses after subtraction of the baseline firing rate (DeYoe and Van Essen 1985). We did not take into account all cells in which the standard error bars of the best and worst responses overlapped (see Burkhalter and Van Essen 1986). In addition, we carried out ANOVA tests to see whether differences in mean cell responses are statistically significant at the level of $P=0.01$. Three repetitions were normally used for each condition, and these were usually enough to make the result significant and allow us to include it into our analyses; when the responses of a cell were not as clear, more repetitions were used (between 5 and 10).

\section{Wavelength selectivity}

To test for the wavelength preference of cells, we used stimuli that were isoluminant for the senior author (Anstis and Cavanagh 1983) and were varied gradually along the blue-green-red, red-yellow-green, red-magenta-blue, or green-cyan-blue axes presented against an equiluminant gray background; the response of the cell to white, black, and gray (control) stimuli was also tested. These tests were also repeated against a black background to avoid possible inhibition of the cell's activity due to the gray background; responses of cells against a black background might be partly due to luminance contrast as well, but because this is kept constant, any color preference can be still spotted out. If a cell showed a preference to a particularly colored stimulus, a series of stationary tests was made during which this preferred color was presented to the receptive field's excitatory center, against a variety of different background colors (including the preferred color), to test for any immediate surround effects. To make sure that any cell preferences were indeed due to the color rather than uncontrolled luminance differences (which should not be the case as isoluminant colors were used), the response of the cell to its preferred color was also compared with the response given to a white bar, and a "white index" was calculated alongside with the color index. The Commission Internationale de l'Eclairage (CIE) $(x, y)$ coordinates for the RGB guns of our monitor were $(0.6047,0.3467),(0.3111,0.5934)$, and $(0.1526,0.0683)$, and so we could use any color inside this color-space triangle to test cells (if in any doubt) in addition to the standardized tests described in the preceding text.

\section{Color-constancy tests}

After the excitatory and inhibitory wavelengths and spatial regions of a particular cell were plotted, we proceeded with the "Mondrian" experiment to test whether a cell's responses correlate with color, as opposed to wavelength. We used a multi-colored display consisting of a series of squares and rectangles (around 20 in total) made of "Color Aid" matt papers. The papers reflected a constant amount of light in all directions to avoid specular effects and were assembled in such a way that none was surrounded by another shape of a single color. The spatial characteristics of the Mondrian were varied for each cell, so that the size (and shape) of the individual Mondrian areas that we used was equal to the size of the excitatory region of the particular cell's receptive field. This was particularly important for cells with a surround inhibited by the same wavelength that excited the center (see RESULTS), and in general ensures that any color constant-like behavior of the cell was not masked by using large monochromatic Mondrian areas. The display was illuminated by three computer-controlled 350W Kodak Carousel projectors, each one having its own light intensity control. Different band-pass filters were used with each one so that the first projector illuminated the display with long-wave light only $(610-700 \mathrm{~nm}$, peak transmittance at $660 \mathrm{~nm})$, the second one with middle-wave light only $(510-570 \mathrm{~nm}$, peak at $530 \mathrm{~nm})$, and the third one with short-wave light only (400-480 nm, peak at $445 \mathrm{~nm}$ ). On several occasions, narrowband interference filters (Ditric Optics) were substituted for the band-pass filters, the three filters having peak transmittances at 630,530 , and $470 \mathrm{~nm}$ and bandwidths at half height of 8-10 nm; the results using either sets of filters were identical. A Gamma Scientific Telephotometer, equipped with an equal energy filter, was used to measure the precise amount of light of any waveband from any area of the display, in radiometric units. Different color areas were put in the receptive field of a cell and each made to reflect various combinations of long-, middle-, and short-wave light. By definition, a "wavelength-selective" cell will respond to any area if there is enough light of its preferred wavelength coming from that area (irrespective of the area's color). A "color-coded" cell (Zeki 1983a), on the other hand, will respond only to areas having a particular color, irrespective of the wavelength combination reflected from them. By using different wavelength combinations and putting different colored areas in the receptive field of the cell, we were able to distinguish between the two. We also tested the effect of the sequence with which the three projectors were turned on: the responses were tested when a cell's receptive field was illuminated first with light of its preferred wavelength, followed by the other two, or in a reverse order, so that in both cases the same triplet of energies was used in the end but via a different temporal route.

\section{Histology}

At the end of the experiment the animals were given a fatal overdose of anesthetic and perfused initially with a solution of $0.9 \%$ saline, followed by $4 \%$ paraformaldehyde in $0.1 \mathrm{M}$ phosphate buffer for a period of about $30 \mathrm{~min}$. The fixative was washed out by perfusing with $1.5110 \%$ phosphate-buffered sucrose, followed by $1.5120 \%$ phosphate-buffered sucrose, followed by $1.5130 \%$ phosphate-buffered sucrose at a flow rate of $50 \mathrm{ml} / \mathrm{min}$. The brain was 
removed from the skull and stored in $30 \%$ buffered sucrose at $4^{\circ} \mathrm{C}$ until it sank. The operculum was dissected out by cutting through the fundi of the lunate, inferior occipital, and calcarine sulci and by making short cuts across the cortex between these sulci. It was flat-mounted on a freezing microtome and sectioned at 50- or $60-\mu \mathrm{m}$ intervals. Sections were stained for cytochrome oxidase (CO) (WongRiley 1979) to visualize the stripe architecture of V2 in relation to the electrode track and thus assign each cell in the penetration to a particular type of stripe.

\section{R E S UL T S}

We have recorded from of 217 cells in dorsal V2 within the representation of paracentral, inferior visual field. Figure 1 shows a typical penetration, near the edge of the lunate sulcus, parallel to the V1/V2 border and thus perpendicular to the cytochrome oxidase stripes. As previously reported (Hubel and Livingstone 1985, 1987; Roe and Ts'o 1995; Shipp and Zeki 1985; Tootell and Hamilton 1989; Ts'o et al. 1990), orientation-selective cells were mostly found in thick stripes and interstripes, a few direction-selective cells in the thick stripe, and a cluster of spectrally tuned cells in one of the thin stripes. In general, when we suspected that we were recording from inside a thin stripe (normally due to the absence of orientation selectivity), we advanced our electrode in smaller steps so as to screen the region more densely for cells with any signs of a spectral tuning. Overall, 69/217 cells have been histologically identified to lie within thin stripes, and 41 of these $(19 \%)$ showed some sort of spectral preference. However, because of the often poor responses to stationary stimuli and the instability of some units, we were able to complete the Mondrian tests for only a total of $27 / 41$ cells; it is the properties of these that are described here. All 27 cells had no orientation or direction preference; 12/27 responded preferentially to long-wave light, $10 / 27$ to middle-wave light, and $5 / 27$ to short-wave light. Although none showed any clear off response to the opponent color flashed inside the receptive field center, 20/27 showed a hidden opponency revealed by a weak response to white light. In addition, 18/27 showed a decrease in response to stimuli larger than the excitatory region of their receptive field, revealing the presence of an inhibitory surround for the same wavelength that selectively activated the center.

Figure 2 shows how the preference of all 217 histologically identified V2 cells recorded from is related to the type of cytochrome-oxidase stripe. As seen in the top diagram, the vast majority of unoriented cells were found in the thin stripes, where we also recorded the larger number of cells responding better to some colors than to others. Color-biased cells were also found in the thick stripes and inter stripes, but the percentage was much smaller and decreased further when more strict classification criteria were used (bottom diagram). Finally, a preference for the direction of motion of the stimulus was rare but nevertheless more often seen in the thick stripes and almost exclusively in the thick-stripes when more strict criteria were used. In summary, these population results support the idea of functional segregation within area V2 and thus reinforced our decision to concentrate on recording from thinstripe cells for our study of the color-specialized system.

Typical responses of all 27 cells are shown in Figs. 3-7. Figure $3 A$ shows a cell with a clear preference for middle-wave light, whereas $B$ reveals that the orientation of the bar used was of no importance, as is the case for the rest of the cells that we selective and biased

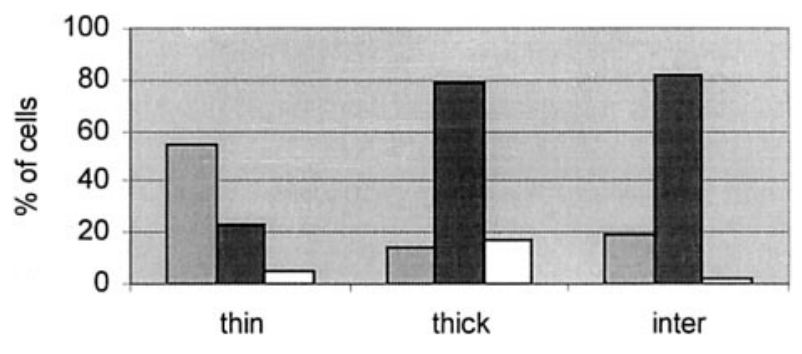

selective only

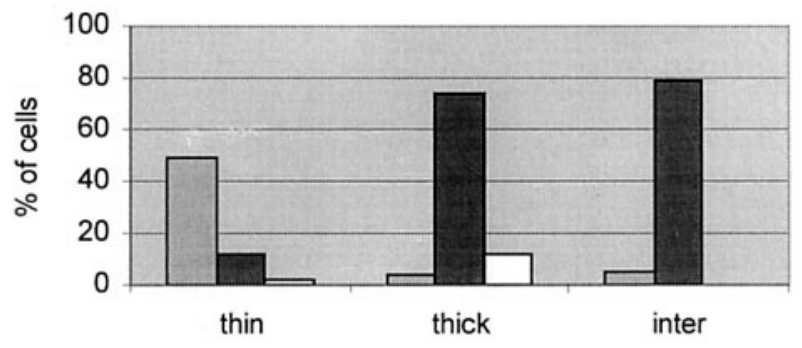

$\square$ colour $\square$ orientation $\square$ direction

FIG. 2. Percentages of cells showing a preference for the color, orientation, or direction of motion of the stimulus in each of the 3 different cytochromeoxidase compartments. The percentages are from a population of 217 histologically identified V2 cells. As in Fig. 1, we used a selectivity index $I=[1-$ (worst-base)/(best-base)], which compares the best and worst responses after subtraction of the baseline firing rate (DeYoe and Van Esssen 1985). We did not take into account cells in which the standard error bars of the best and worst responses overlapped (see Burkhalter and Van Essen 1986). Top: cells with a selectivity index greater or equal to 0.5 ; bottom: diagram cells with a selectivity index greater or equal to 0.7 .

report here. The presence of a suppressive surround for the same (middle) wavelength which preferably excites the center is revealed in Fig. $3 C$ : the cell does not respond well to small stimuli, gives a maximal response when the size of the stimulus is equal to the size of the excitatory region of the receptive field, but decreases its response when the stimulus size is further increased. When studying such cells, care was taken that in the Mondrian tests, the cell's excitatory receptive field was not surrounded by areas with a high reflectance for the preferred wavelength, to obtain a maximal response from the cell. Figure $4 A$ shows the responses of the same cell to Mondrian areas of different color made to reflect the identical triplet of wavelengths of which the middle one was the dominant. The cell responds well to all areas regardless of their actual color; a slightly weaker response to the red area is due to the difficulty of making this area reflect large amounts of middle-wave light. Such cells, which respond to any area of the Mondrian as long as it reflects a certain wavelength combination, can also be made not to respond to their preferred Mondrian area if it does not reflect a sufficient amount of their preferred wavelength. This is illustrated in Fig. $4 B$, showing the responses of another middle-wavelength-selective cell when a green area of the Mondrian was placed inside its receptive field and illuminated with two different energy triplets: it responded strongly when the area was made to reflect more middle than short- and 

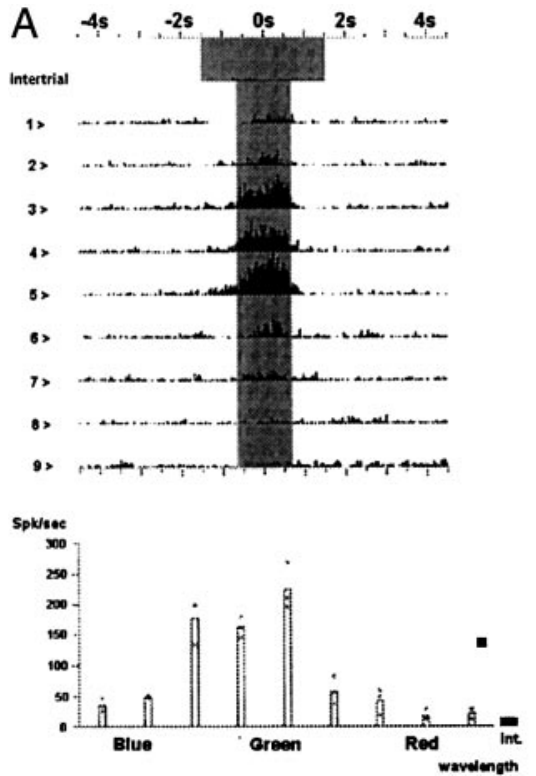
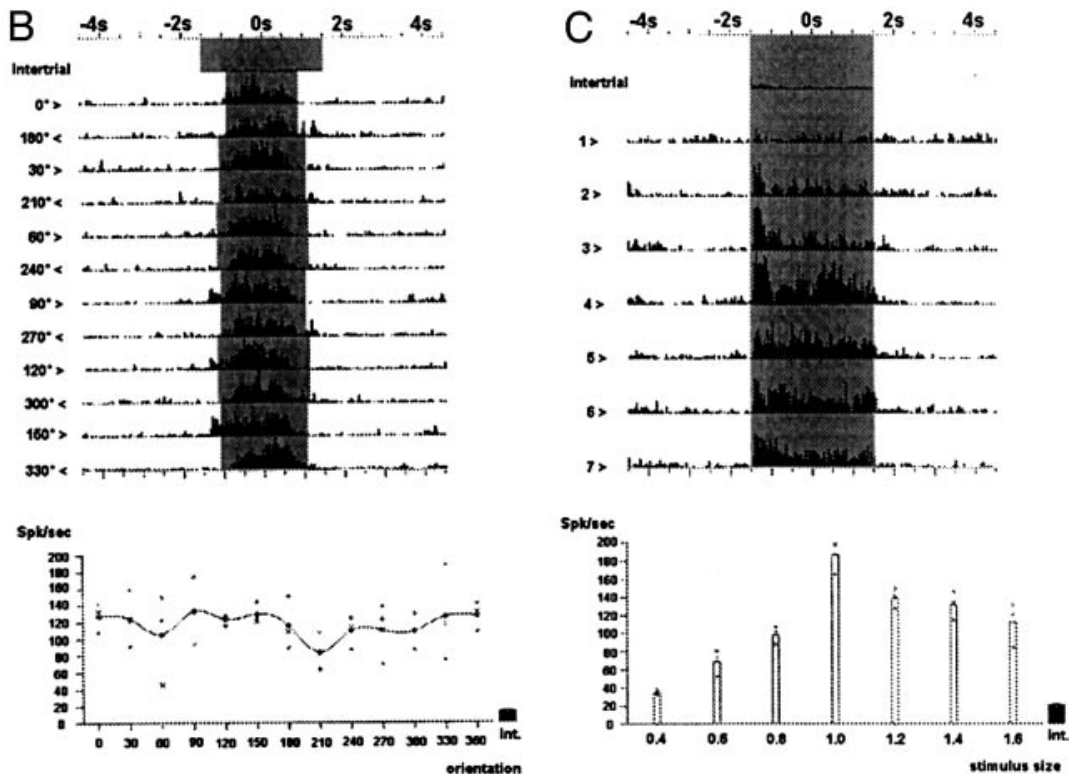

FIG. 3. Average responses of a V2 cell to 3 -s on-screen stimuli (between -1.5 and $+1.5 \mathrm{~s}$ ) repeated 3 times. $\square$, the time during which the stimulus was in the cell's receptive field. Cell responses are calculated during this epoch. Trials were randomly intermixed, with a 3-s intertrial period during which the screen was blank (top). A: responses to isoluminant vertical bars of different colors across the spectrum, moving against a black background. Commission Internationale de l'Eclairage (CIE) coordinates $(x, y)$ of the 9 colors (from left to right): $(0.1525,0.0682)(0.2220,0.2992)(0.2498,0.3896)(0.2814,0.4937)(0.3112,0.5935)$ $(0.4627,0.4660)(0.5096,0.4269)(0.5742,0.3711)(0.6043,0.3460)$. B: responses to green moving bars of various orientations against a black background. $C$ : responses to stationary green squares of varying size (given as a percentage of the area of the excitatory receptive field center) against a black background.

long-wave light (triplet A), but much less when it was made to reflect more short- and long-wave light than middle (triplet B), although it looked green to a normal observer in both conditions. Normally we tested each cell using three to four different illumination triplets and another three to four different areas of the Mondrian.

The preceding characteristics are typical of all $27 \mathrm{~V} 2$ cells encountered in this study: their responses did not correlate with color but were dependent on wavelength composition alone. Figure 5 shows the results of a quantitative comparison of the effect of changing the wavelength composition of the illumination to that of changing the color of the area inside the cells' receptive field. The ratio between the best and worst responses is calculated for each cell, both for different illuminations, and for different colors. Straight lines at 1.5 divide the diagram into four regions; all 27 cells fall within the lower-right quadrant, indicating that the modulatory effect of the illumination composition on cell responses is strong and that of color is weak. A typical color-coded cell ignoring changes in illumination and only signaling changes in natural color (Zeki 1983a) should fall in the upper-left quadrant, but none of our cells in V2 do.

In addition to wavelength composition, the sequence with which the three projectors were switched on was also a determining factor for all cells. Figure 6 shows the responses of one long- and one middle-wave-selective cell to different sequences of illumination of the red and the green area of the Mondrian respectively. When the projector of the preferred wavelength was switched on first, they gave a strong response, which was abolished when the other two projectors were added. When the two other projectors were switched on first, the cells did not respond; but when the projector of the preferred wavelength was added to the other two, the cells gave a strong response. These cells thus respond only if the light of the preferred wavelength is added to the other two lights: although the final energy triplet illuminating the Mondrian was the same in both cases, the cells only responded in the second case. Therefore the responses of such cells, as well as depending on wavelength composition, also signal a change in the amount of the cell's preferred wavelength with respect to the other wavelengths, the cell being excited when there is an increase and inhibited when there is a decrease. Figure 7 also illustrates both the dependence of the response of another cell to the wavelength composition as well as the sequence of illumination. This cell responded to a blue and a red area of the Mondrian reflecting the same triplet of wavelengths, the predominant of which was the short one. If, however, instead of all three projectors being switched on together at the same time, the long- and middle-wave projectors were switched on as a pair, separately from the short-wave projector, the cell would or would not respond to the same energy triplet depending on whether there was an increase or decrease in the amount of its preferred wavelength with relative to the other two.

Could this response dependency on the temporal ordering of stimulation be simply accounted for by classical chromatic adaptation? For example, in Fig. 6A, one might argue that when the middle-short-wave light (MS) stimulus is presented first for $1 \mathrm{~s}$, the MS system adapts and cannot exhibit its inhibitory effect on the long-wave-light (L) signal, which thus evokes a response from the cell-unlike what happens when the MS stimulus follows the L one. This is an unlikely possibility since when all three projectors are switched on together the cell responds (because the red area illuminated by white light reflects more L than MS light) and therefore no inhibitory effect due to the MS stimulus is evident under these conditions. Another possibility could be that the L systems adapts and thus the "inhibitory" effect when the MS stimulus comes on. There 
A
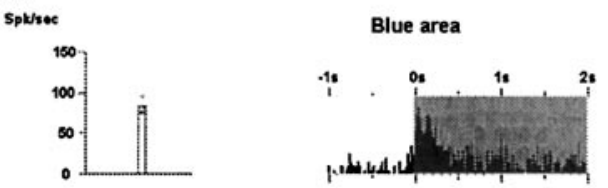

Spks:
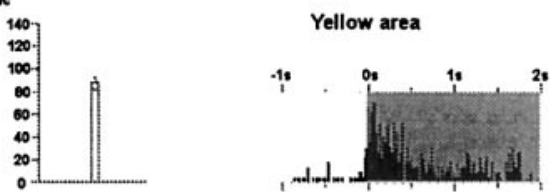

Splunec
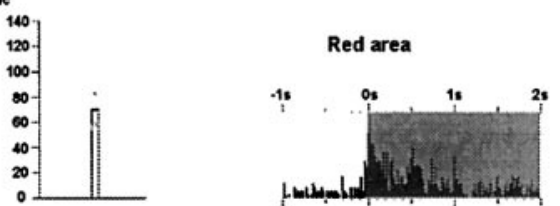

B
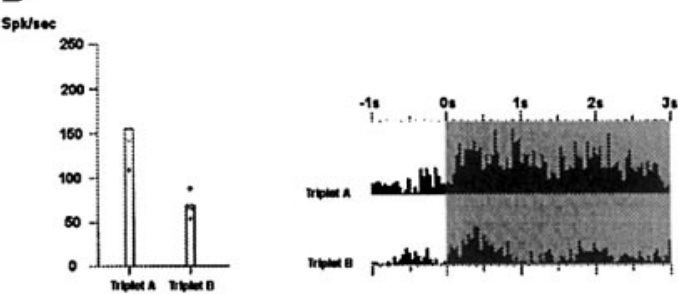

FIG. 4. Mondrian test for distinguishing between wavelength composition and color; shaded area shows time of stimulation. A: the cell of Fig. 2 gave the same response to different color patches illuminated in such a way as to reflect 40,80 , and $20 \mathrm{~mW} * \mathrm{Sr}^{-1} * \mathrm{~m}^{-2}$ of long-, middle-, and short-wave light, respectively (a suspected slightly higher response to yellow did not survive the ANOVA test). This cell thus responded to any area of the Mondrian made to reflect a predominant amount of middle wavelength. $B$ : responses of another middle-wave selective cell under 2 different types of illumination of a green patch. Although the patch appeared green in both cases, the cell responded much stronger when there was an abundance of middle-wave light reflected from it (triplet A: 30,60 , and $15 \mathrm{~mW} * \mathrm{Sr}^{-1} * \mathrm{~m}^{-2}$; triplet $\mathrm{B}: 40,30$, and 30 $\left.\mathrm{mW} * \mathrm{Sr}^{-1} * \mathrm{~m}^{-2}\right)$.

are no such signs of adaptation, however; and the cell in both cases responds vigorously to red light during the whole $1 \mathrm{~s}$ of L stimulation. Similarly, it is unlikely that we have MS adaptation within $1 \mathrm{~s}$, otherwise the inhibitory effect in Fig. 6 A (top) should not last for so long [unless the L system, which showed no sign of adaptation during the 1st second, also begins to adapt at exactly the correct time and rate so that the total long-middle-short-wave light (LMS) output remains 0 -an almost impossible situation]. In fact, there are no signs of adaptation in Fig. 7 either, where stimulation lasts 2 rather than $1 \mathrm{~s}$, or even in Fig. $6 B$ (with the exception of the LMS stimulus), where stimuli are $3 \mathrm{~s}$ long. It is therefore the detection of illumination changes rather than classical color-adaptation that is responsible for the behavior of these cells.

\section{I S C U S S I O N}

In our search for any sort of chromatic tuning, we have recorded from and histologically identified (with respect to cytochrome-oxidase stripes) a total number of $217 \mathrm{~V} 2$ cells. Our global results verify previous reports of a functional seg- regation within this area, related to its characteristic cytochrome-oxidase pattern (see INTRODUCTION). It is perhaps worth mentioning that although in most cases we recorded from single units, in some cases, we might have recorded from more than one neuron. In the latter case, tuning curves to different visual attributes would represent the average from a number of neurons with possible different selectivities, giving perhaps a broader tuning curve. We believe, however, that this did not bias our global results in any way mainly because of the rarity of such occasions. Furthermore, the properties of neurons change gradually as one advances the electrode through cortex (preferred orientation, for example), and nearby cells normally share the same (or very similar) type of selectivity. Therefore even when we were not able to isolate single units, averaging between neighboring units would not have significantly distorted the tuning characteristics of the neuron(s). Also, an artifact of broader tuning curves (i.e., less selectivity) would tend to bias the results toward a picture of less segregation within V2. The fact that, despite any hypothetical artifact working against us, we were still able to report significant segregation, emphasizes that cells with different selectivities are segregated within the cortex of area V2.

Because V2 receives its input mainly from V1, including subdivisions of V1 specialized for color processing (Livingstone and Hubel 1984), the presence of color-selective cells in this area should be expected. Indeed, in early studies, $16 \%$ of the cells in the posterior bank of the lunate sulcus of both the anesthetized and the awake behaving monkey were found to respond with excitation to some colors and inhibition to others

\section{best/worst}

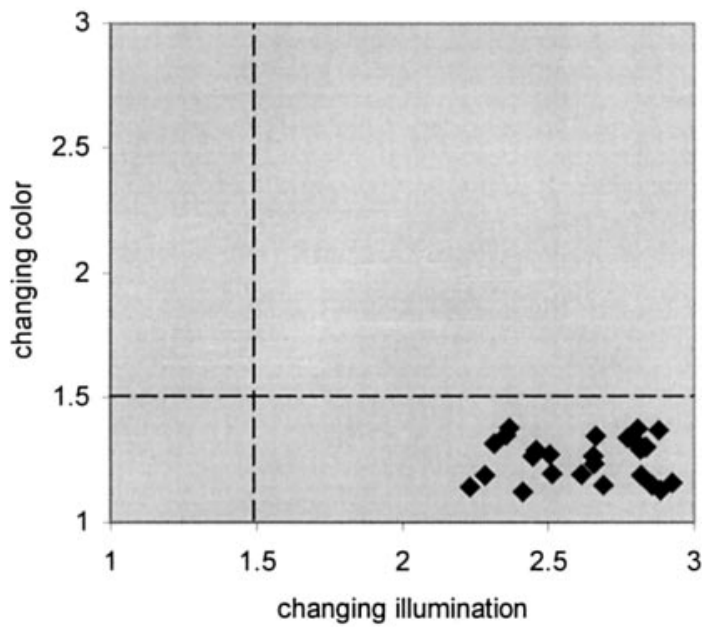

FIG. 5. Group quantitative results of the effect of changing the wavelength composition and the area of the Mondrian on cell responses. Two indices are calculated for each neuron: a color index equal to the ratio between the best and worst response of the cell to Mondrian areas of different colors made to reflect the cell's preferred illumination triplet, and a wavelength index equal to the ratio between the best and worst response of the cell to different triplets of illumination reflected by the area of its preferred color. A ratio more than 1.5 is arbitrarily taken to indicate a significant influence of the changing factor (wavelength/color) on the response of the cell. The diagram shows that responses are much more strongly modulated by changes in the wavelength composition of the illuminating light than by changes in the color. As well as using the indices to measure the size of the effect, ANOVA tests show that the differences between best and worst mean firing rates is statistically significant when changing the wavelength composition of the illumination but not when changing the color of the target Mondrian area. 
A sonsons

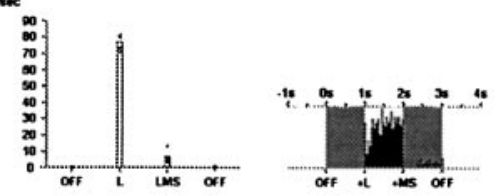

$-$

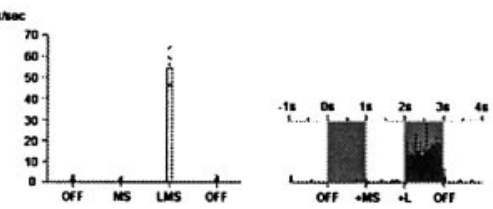

B
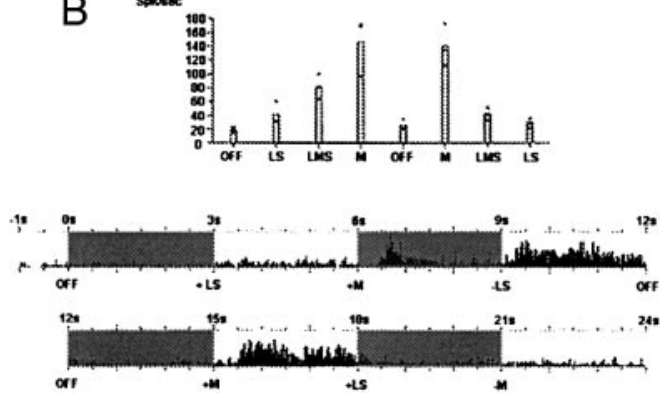

FIG. 6. The responses of a V2 cell to different sequences of illumination of a blue $(A)$ and a red $(B)$ area of the Mondrian, reflecting the same final triplet of energies $\left(30,20\right.$, and $\left.50 \mathrm{~mW} * \mathrm{Sr}^{-1} * \mathrm{~m}^{-2}\right)$. Left: the cell's preferred wavelength (short) was added to the other 2, whereas the opposite is true for the right column. There was no time lag between the long-middle-wave light (LM) or short-wave light (S) and long-middle-short-wave light (LMS) conditionsthe vertical separation of the initial and final stimuli in the figure is for illustrative purposes only (i.e., time $0 \mathrm{~s}$ for the LMS stimulation is equal to time $2 \mathrm{~s}$ of the LM or $\mathrm{S}$ stimulation).

(Baizer et al. 1977; Zeki 1978b); all gave spatially coextensive color opponent responses in a round or oval center, and some had in addition suppressive surrounds that limited the size of a stimulus effective in eliciting one or both of the center responses (Baizer et al. 1977). These results were confirmed by later studies (Burkhalter and Van Essen 1986; Gegenfurtner et al. 1996; Hubel and Livingstone 1985, 1987; Kiper et al. 1997; Levitt et al. 1994), although the exact percentage of color cells in V2 as well as the spatial receptive field organization varied across publications from different laboratories. In the present study, we report on cells that are preferentially excited by stimuli of a particular wavelength, some of which also have a suppressive surround for the same wavelength that excites the center (Baizer et al. 1977). Our stimuli were not designed to map the exact spatial extent and configuration of these surrounds nor to accurately describe any excitatory surround effects (Livingstone and Hubel 1987). In some cases, center opponency was detected as a much weaker response to white stimuli compared with stimulation with the preferred color. The purpose of our initial, stereotype, tests was not a full description of receptive field organization but to screen cells for any differential responses across the spectrum (and also for any inhibitory surround) before moving on to the Mondrian tests, which were the main aim of this experiment.

All V2 cells we have recorded from respond to the wavelength composition rather than the color of the stimulus, suggesting that color constancy is not achieved before reaching area V4 in the color pathway. This is in agreement with lesion studies, which show that color constancy is lost after removal of V4, whereas wavelength discrimination is not, probably due to the integrity of areas V1 and V2, in both man (Kennard et al. 1995; Vaina 1994) and monkey (Heywood et al. 1992; Walsh et al. 1992, 1993; Wild et al. 1985). The method we have used in this study is similar to that used by Zeki (1983a) in areas V1 and V4. Its capability to distinguish between color-coded and wavelength-selective cells has been recently confirmed by our studies in area V4 of the awake behaving monkey using a different paradigm (Kusunoki et al. 2001). Concerning the present study, although we have had the additional advantage of quantifying our computerized data, it is still possible that this method is not sufficiently sensitive to detect and describe in detail more subtle modulations of cell responses as a function of perceptual changes. Previous studies have described some subcortical color-"constant-like" behavior (Creutzfeldt et al. 1991a,b), where neurons could modulate their firing rate by stimulation of the surround. These experiments, however, instead of using multicolored Mondrian-type surrounds illuminated together with the central stimulus, involved uniform monochromatic surrounds presented for long periods of time. They are therefore more appropriate for specifically studying the effects of color contrast and color adaptation rather than the phenomenon of color constancy (the relation of which to contrast and adaptation is still a matter of dispute) as a whole. A cell behavior similar to the one reported in these subcortical studies has also been reported in striate cortex (Wachtler et al. 1999), using similar methods, but no firm conclusions can be drawn because the relevant study is only available in abstract
A

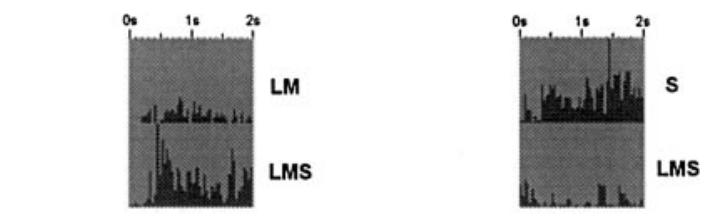

Average histograms. Bar width $40 \mathrm{~ms}$
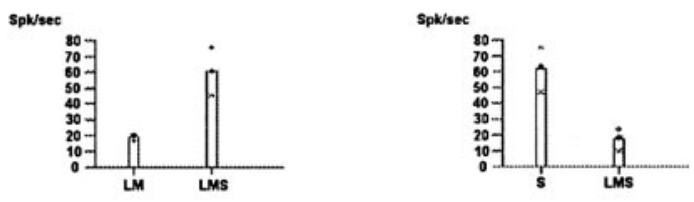

B

Average histograms. Bar width $40 \mathrm{~ms}$
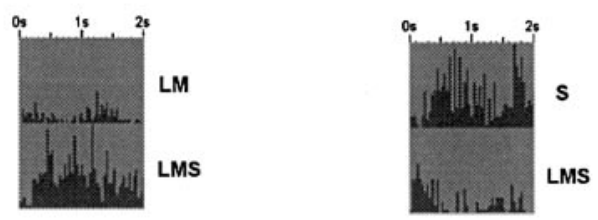

Spk/sec
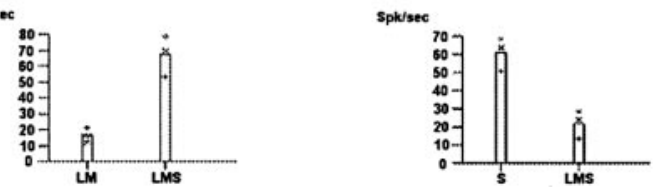

FIG. 7. The responses of $2 \mathrm{~V} 2$ cells, 1 selective for long-wave light $(A)$ and the other for middle-wave light $(B)$ to different sequences of illumination of Mondrian areas having their preferred color (red and green, respectively) with white light (LMS condition, where L, long-wave light; M, middle-wave light; and $\mathrm{S}$, short-wave light). Both cells gave a different response to the same energy triplet depending on the temporal route of stimulation. 
form. Concerning achromatic (brightness) interactions, modulation of the firing rate of neurons due to uniform changes in illumination outside the classical receptive field have been reported in both LGN and striate cortex, but not in the retina (Rossi and Paradiso 1999). To our knowledge, this is the first study of color constancy in area V2. Although initially it might seem contradictory, the lack of any "color-coded" cells in this area is consistent with recent psychophysical evidence showing that although spatial chromatic interactions take place very early in the visual system, the generation of color per se is a property of higher visual areas of the brain (Moutoussis and Zeki 2000).

We also describe here for the first time V2 cells performing a temporal comparison as well, signaling a relative increase or decrease in the amount of their preferred wavelength reflected from an area inside their receptive field. The brief presentation times that we used, together with the temporal characteristics of the responses of our cells (see RESULTS) excludes the possibility of classical color adaptation being an explanation of this behavior. It is well known that chromatic adaptation can influence color appearance in humans (Brindley 1953; Stiles 1959) and the responses of cells early in the monkey visual pathway (Creutzfeldt et al. 1991a,b; DeMonasterio and Gouras 1977; DeMonasterio et al. 1975) and that postreceptoral (contrast) adaptation can influence both color appearance and color constancy (Webster and Mollon 1991, 1995). For such effects to manifest themselves, however, presentation of the adapting stimuli needs to be longer than the presentation times we used in our "temporal signalling" experiments. Furthermore, we have not observed any adaptation-like behavior in the responses of our cells: both excitatory and inhibitory effects remained unchanged during the whole period of our (brief) stimulation. Similar temporal response properties to the ones we report here in V2 have been also reported in V1 (Zeki 1983 b), but their role in an invariant, Mondrian-type situation is not clear at first sight. However, the fact that the wavelength composition coming from objects in the real world is continuously changing suggests that the brain could use the information that these cells provide to compensate for temporal illumination changes taking place in natural viewing conditions. Such cells, together with cells that have an inhibitory surround for the same wavelength that selectively excites their center, could contribute to brain color constancy mechanisms by performing a continuous wavelength comparison across space and time.

The cells we have recorded from were not uniformly sampled from area V2. We have chosen to concentrate on cells from the thin cytochrome-oxidase stripes, which showed no selectivity for the orientation of the stimulus, because we believe them to belong to the V2 part of the color-specialized system of the visual brain (Baizer et al. 1977; DeYoe and Van Essen 1985; Felleman et al. 1997; Hubel and Livingstone 1985, 1987; Malach et al. 1994; Roe and Ts'o 1995; Shipp and Zeki 1985; Tootell and Hamilton 1989; Ts'o et al. 1990; Xiao et al. 1999; Zeki and Shipp 1989). Spectrally tuned cells can also be found outside thin stripes, but there is no obligatory 1:1 relationship between properties at the cellular level and perceptual attributes: color signals in thin stripes may subserve color vision per se (i.e., determining surface reflectance properties), whereas color signals outside thin stripes may be relevant to the determination of surface contours, depths and directions of motion, etc. Furthermore, these were the cells that showed a clear-cut preference for a particular wavelength and also gave good repetitive responses to long periods of testing with stationary Mondrian-type stimuli. By showing that such cells are heavily concentrated within the thin stripes of $\mathrm{V} 2$, we both confirmed past physiological studies but also fortified our belief in the general strategy of functional specialization in the visual brain.

In summary, the results of this study show that the "color" cells of V2 are in fact wavelength specific, responsive to the wavelength composition of light, and indifferent to the color of a patch in their receptive fields. We were not able to find in V2 any cells with the color-constant properties found in V4 of both the anesthetized (Zeki 1983a) and the awake behaving monkey (Kusunoki et al. 2001). We cannot exclude the possibility that such cells may nevertheless exist in V2. If so, their demonstration will require the development of more sensitive methods than the ones we have used here.

We thank J. Romaya for the computer programming.

This work was supported by the Wellcome Trust.

\section{REFERENCES}

AlLMAN JM AND KAAS JH. Representation of the visual field in striate and adjoining cortex of the owl monkey (Aotus trivirgatus). Brain Res 35: 89-106, 1971.

Anstis S and Cavanagh P. A minimum motion technique for judging equiluminance. In: Colour Vision, Physiology and Psychophysics, edited by Mollon JD and Sharpe LT. London, UK: Academic, 1983, p. 155-166.

BAIZER JS, RoBINSON DL, AND Dow BM. Visual responses of area 18 neurons in awake, behaving monkey. J Neurophysiol 40: 1024-1037, 1977.

BARTELS A AND ZEKI S. The architecture of the color center in the human visual brain. Eur J Neurosci 12: 172-193, 2000.

Born RT AND ToOtell RB. Spatial frequency tuning of single units in macaque supragranular striate cortex. Proc Natl Acad Sci USA 88: 70667070, 1991

BRINDLEY GS. The effects on colour vision of adaptation to very bright lights. J Physiol (Lond) 122: 332-350, 1953.

BurkHALTER A AND VAN ESSEN DC. Processing of color, form and disparity information in visual areas VP and V2 of ventral extrastriate cortex in the macaque monkey. J Neurosci 6: 2327-2351, 1986.

CRAGG BG. The topography of the afferent projections in circumstriate visual cortex studied by the Nauta method. Vision Res 9: 733-747, 1969.

Creutzfeldt OD, Crook JM, Kastner S, Li CY, and Pei X. The neurophysiological correlates of color and brightness contrast in lateral geniculate neurons. I. Population analysis. Exp Brain Res 87: 3-21, 1991a.

Creutzfeldt OD, Kastner S, Pei X, and Valberg A. The neurophysiological correlates of color and brightness contrast in lateral geniculate neurons. II. Adaptation and surround effects. Exp Brain Res 87: 22-45, 1991 b.

De Monasterio FM AND Gouras P. Responses of macaque ganglion cells to far violet lights. Vision Res 17: 1147-1156, 1977.

De Monasterio FM, Gouras P, and Tolhurst DJ. Concealed color opponency in ganglion cells of the rhesus monkey retina. J Physiol (Lond) 251: 217-229, 1975.

DeYoe EA, Felleman DJ, Van Essen DC, and McClendon E. Multiple processing streams in occipitotemporal visual cortex. Nature 371: 151-154, 1994.

DeYoe EA And VAn Essen DC. Segregation of efferent connections and receptive field properties in visual area V2 of the macaque. Nature 317: $58-61,1985$

FELlEMAN DJ, XIAO Y, AND MCCLENDON E. Modular organization of occipitotemporal pathways: cortical connections between visual area 4 and visual area 2 and posterior inferotemporal ventral area in macaque monkeys. J Neurosci 17: 3185-3200, 1997.

Gegenfurtner KR, Kiper DC, and Fenstemaker SB. Processing of color, form, and motion in macaque area V2. Vis Neurosci 13: 161-172, 1996.

Heywood CA, Gadotti A, AND Cowey A. Cortical area V4 and its role in the perception of color. J Neurosci 12: 4056-4065, 1992. 
Hubel DH AND Livingstone MS. Complex-unoriented cells in a subregion of primate area 18. Nature 315: 325-327, 1985.

Hubel DH AND LivingSTONE MS. Segregation of form, color, and stereopsis in primate area 18. J Neurosci 7: 3378-3415, 1987.

Kennard C, LAwden M, MoRland AB, AND Ruddock KH. Color identification and color constancy are impaired in a patient with incomplete achromatopsia associated with prestriate cortical lesions. Proc R Soc Lond B Biol Sci 260: 169-175, 1995.

KIPER DC, Fenstemaker SB, AND Gegenfurtner KR. Chromatic properties of neurons in macaque area V2. Vis Neurosci 14: 1061-1072, 1997.

Kusunoki M, Moutoussis K, AND ZEKI S. The effect of background on the colour selective cells in area V4 of monkeys. Soc Neurosci Abstr 27: 286.13, 2001.

LAND EH. The retinex theory of color vision. Proc Roy Inst GB 49: 23-58, 1974.

Land EH and McCann JJ. Lightness and retinex theory. J Opt Soc Am 61: $1-11,1971$.

LEVITT JB, KIPER DC, AND Movshon JA. Receptive fields and functional architecture of macaque V2. J Neurophysiol 71: 2517-2542, 1994.

LivingSTONE MS AND HuBEL DH. Thalamic inputs to cytochrome oxidase-rich regions in monkey visual cortex. Proc Natl Acad Sci USA 79: 6098-6101, 1982.

Livingstone MS AND HuBEL DH. Specificity of cortico-cortical connections in monkey visual system. Nature 304: 531-534, 1983.

LiVINGSTONE MS AND Hubel DH. Anatomy and physiology of a color system in the primate visual cortex. $J$ Neurosci 4: 309-356, 1984.

Malach R, Tootell RB, and MaloneK D. Relationship between orientation domains, cytochrome oxidase stripes, and intrinsic horizontal connections in squirrel monkey area V2. Cereb Cortex 4: 151-165, 1994.

MERriLl EG AND Ainsworth A. Glass-coated platinum-plated tungsten microelectrodes. Med Biol Eng 10: 662-672, 1972.

Moutoussis K AND ZEKI S. A direct demonstration of perceptual asynchrony in vision. Proc R Soc Lond B Biol Sci 264: 393-399, 1997a.

Moutoussis K AND ZeKI S. Functional segregation and temporal hierarchy of the visual perceptive systems. Proc R Soc Lond B Biol Sci 264: 1407-1414, 1997b.

Moutoussis K AND ZEKI S. A psychophysical dissection of the brain sites involved in color-generating comparisons. Proc Natl Acad Sci USA 97: 8069-8074, 2000.

NaKamura H, Gattass R, Desimone R, and Ungerleider LG. The modular organization of projections from areas V1 and V2 to areas V4 and TEO in macaques. J Neurosci 13: 3681-3691, 1993.

RoE AW AND Ts'o DY. Visual topography in primate V2: multiple representation across functional stripes. J Neurosci 15: 3689-3715, 1995.

Rossi F AND PARADISO MA. Neural correlates of perceived brightness in the retina. Lateral geniculate nucleus, and striate cortex. J Neurosci 19: 61456156, 1999.

SCHILLER PH. On the specificity of neurons and visual areas. Behav Brain Res 76: 21-35, 1996.

SCHILlER PH. Past and present ideas about how the visual scene is analysed by the brain. Cereb Cortex 12: 59-90, 1997.

Sereno Mi, Dale AM, Reppas JB, Kwong KK, Belliveau JW, Brady TJ, Rossen BR, AND TOOTELL RBH. Borders of multiple visual areas in humans revealed by functional magnetic resonance imaging. Science 268: 889-893, 1995.

ShipP S, Blanton M, AND ZEKi S. A visuo-somatomotor pathway through superior parietal cortex in the macaque monkey: cortical connections of areas V6 and V6A. Eur J Neurosci 10: 3171-3193, 1998.

Shipp S, Watson JDG, Frackowiak RSJ, and ZeKi S. Retinotopic maps in human prestriate visual cortex: the demarcation of areas V2 and V3. Neurolmage 2: 125-132, 1995.
SHIPP S AND ZEKI S. Segregation of pathways leading from area V2 to areas V4 and V5 of macaque monkey visual cortex. Nature 315: 322-325, 1985.

STILES WS. Color vision: the approach through increment threshold sensitivity. Proc Natl Acad Sci USA 45: 100-114, 1959.

Tootell RB AND Hamilton SL. Functional anatomy of the second visual area (V2) in the macaque. J Neurosci 9: 2620-2644, 1989.

Tootell RB, Silverman MS, De Valois RL, and Jacobs GH. Functional organization of the second cortical visual area in primates. Science 220: 737-739, 1983.

Tootell RB, Silverman MS, Hamilton SL, De Valois RL, and Switkes E. Functional anatomy of macaque striate cortex. III. Color. J Neurosci 8: 1569-1593, 1988

Ts'o DY, Frostig RD, Lieke EE, AND Grinvald A. Functional organization of primate visual cortex revealed by high resolution optical imaging. Science 249: 417-420, 1990

Ts'o DY AND GILBERT CD. The organization of chromatic and spatial interactions in the primate striate cortex. J Neurosci 8: 1712-1727, 1988.

VAINA LM. Functional segregation of color and motion processing in the human visual cortex: clinical evidence. Cereb Cortex 4: 555-572, 1994.

WACHTLER T, SEJNOWSKI TJ, AND AlBRIGHT TD. Interactions between stimulus and background chromaticities in macaque primary visual cortex (Abstract). IOVS 40: 3378-B236, 1999.

Walsh V, Carden D, Butler SR, and Kulikowski JJ. The effects of V4 lesions on the visual abilities of macaques: hue discrimination and color constancy. Behav Brain Res 53: 51-62, 1993.

WAlsh V, Kulikowski JJ, ButLer SR, AND CARDEN D. The effects of lesions of area V4 on the visual abilities of macaques: color categorization. Behav Brain Res 52: 81-89, 1992.

Webster MA AND Mollon JD. Changes in color appearance following post-receptoral adaptation. Nature 349: 235-238, 1991.

WEBSTER MA AND MoLLON JD. Color constancy influenced by contrast adaptation. Nature 373: 694-698, 1995.

Wild HM, Butler SR, Carden D, and Kulikowski JJ. Primate cortical area V4 important for color constancy but not wavelength discrimination. Nature 313: 133-135, 1985.

Wong-RILEY M. Changes in the visual system of monocularly sutured or enucleated cats demonstrable with cytochrome oxidase histochemistry. Brain Res 171: 11-28, 1979.

Xiao Y, Zych A, AND Felleman DJ. Segregation and convergence of functionally defined V2 thin stripe and interstripe compartment projections to area V4 of macaques. Cereb Cortex 9: 792-804, 1999.

ZEKI S. Representation of central visual fields in prestriate cortex of monkey. Brain Res 14: 271-291, 1969.

ZEKI S. Colour coding in rhesus monkey prestriate cortex. Brain Res 53: 422-427, 1973.

ZEKI S. Colour coding in the superior temporal sulcus of rhesus monkey visual cortex. Proc R Soc Lond B Biol Sci 197: 195-223, 1977.

ZEKI S. Functional specialisation in the visual cortex of the rhesus monkey. Nature 274: 423-428, 1978a.

ZEKI S. Uniformity and diversity of structure and function in rhesus monkey prestriate visual cortex. J Physiol (Lond) 277: 273-290, 1978b.

ZEKI S. Colour coding in the cerebral cortex: the reaction of cells in monkey visual cortex to wavelengths and colors. Neuroscience 9: 741-765, 1983a.

ZEKI S. Colour coding in the cerebral cortex: the responses of wavelengthselective and color-coded cells in monkey visual cortex to changes in wavelength composition. Neuroscience 9: 767-781, 1983b.

ZEKI S. Localization and globalization in conscious vision. Annu Rev Neurosci 24: 57-86, 2001

ZEKI S AND SHIPP S. Modular connections between areas V2 and V4 of macaque monkey visual cortex. Eur J Neurosci 1: 494-506, 1989. 